\title{
Femtosecond and picosecond near-field ablation of gold nanotriangles: nanostructuring and nanomelting
}

\author{
A. Kolloch - T. Geldhauser - K. Ueno - H. Misawa • \\ J. Boneberg · A. Plech · P. Leiderer
}

\begin{abstract}
We report on recent insights into the interaction between ultra-fast laser pulses and plasmonic nanoparticles. We discuss femtosecond near-field ablation as a simple but versatile tool for the nanoscale modification of surfaces and the high-resolution measurement of a nanostructure's near field. Two model systems are presented, illustrating the complexity of near-field distributions. Furthermore, finite difference time domain calculations in combination with absorption spectra provide a deeper insight into the factors influencing the near-field distribution. For the first time, an almost perfect agreement between the measured ablation pattern and experiment has been reached for gold triangles with a side length around $500 \mathrm{~nm}$. Additionally, the results from picosecond laser irradiated plasmonic structures display a new regime of nanoscale laser material processing. We present first results showing nanometre confined melting induced by laser pulses.
\end{abstract}

\section{Introduction}

Focusing light down to the nanometre scale is important for a wide range of applications from the local modification of a substrate [1] to highly effective optical pumping

A. Kolloch (®) $\cdot$ J. Boneberg $\cdot$ P. Leiderer

University of Konstanz, Konstanz, Germany

e-mail: andreas.kolloch@uni-konstanz.de

T. Geldhauser $\cdot$ K. Ueno $\cdot$ H. Misawa

Research Institute of Electronic Science, Hokkaido University, Sapporo, Japan

A. Plech

Karlsruhe Institut für Technologie, Karlsruhe, Germany of nanoscopic structures (e.g. quantum dots [2] and molecules [3]).

A promising candidate for this goal is the optical near field of micro- and nanostructures with its highly localised electromagnetic field distribution, which allows for focusing down to a few nanometres. Though several applications for this local field enhancement have been successfully demonstrated [2-6], the direct measurement of the local field distribution is still of great importance for the understanding and most efficient application of the optical near field.

As an alternative to optical scanning techniques (e.g. scanning near-field optical microscopy (SNOM) [7]), nearfield lithography methods have been proposed as highresolution methods measuring the optical near-field distribution of nanostructures. These techniques use the strong field enhancement of the optical near field to locally modify an imaging layer beneath the structure $[8,9]$, a polymer matrix around the structure [10-12] or the surface beneath a nanostructure itself $[1,8,13-15]$, leaving a permanent imprint of the field distribution with a single laser pulse. Thereafter, the modified surface can be examined using high-resolution microscopy.

In this article, we report on recent developments in direct near-field lithography by means of femtosecond ( $f s$ ) as well as picosecond (ps) laser pulses and discuss the factors determining the local field distribution of a given nanostructure. Furthermore, we will present calculations that reproduce the measured ablation pattern almost perfectly and provide a deeper insight into the relation between shape and local field distribution of the plasmon resonance of a given nanostructure.

Finally, we will discuss the differences between the clearly thermally driven ablation in the ps time regime and the fs ablation experiments. 


\section{Sample preparation}

All metallic nanostructures discussed in the following were prepared using shadow masks composed of a monolayer of colloidal particles. To form this monolayer, we used a modified version of the monolayer process, as proposed by Burmeister et al. [16] and others [17-21].

The colloids were adsorbed onto a water/air surface. Due to their mobility on this surface, the particles self organise into a closely packed layer. In this closely packed form, the resulting two-dimensional colloidal crystal displays a sixfold symmetry with triangular holes between the particles.

Slowly removing the water from below the particles, the monolayer can be cast on any given substrate. In this study, we used silicon substrates (crystal orientation: [111], pdoped, resistivity: 1-2 $\Omega \mathrm{cm}$ ) due to their low surface roughness and environmental stability.

After deposition of the monolayer, a gold film (thickness $40 \mathrm{~nm}$ ) was evaporated onto the samples. As the gold can only reach the silicon surface through the triangular holes between the colloids, a pattern of gold triangles forms on the surface. After removing the colloids from the surface, triangular structures with very sharp tips (down to $5 \mathrm{~nm}$ tip radius) are obtained, something not easily achieved by conventional lithography methods [13].

\section{Experiment and calculations}

For the fs ablation experiments, a single intense fs laser pulse $(800 \mathrm{~nm}, 150 \mathrm{fs}$, up to $10 \mathrm{~mJ})$ from a multipass Ti:Sapphire (Ti:Sa) amplifier system (Spectra Physics) was directed onto the sample at an angle of incidence of $35^{\circ}$. The polarisation of the laser pulse was perpendicular to the plane of incidence, providing a parallel polarisation with respect to the sample surface.

The sample was mounted below a video microscope on a micrometre bench giving control over the exact spot illuminated by the laser.

For the ps ablation experiments, we used an improved version of the setup in which the incident laser pulse was normal to the sample surface. The picosecond laser pulse was generated by using the aforementioned laser system without the final pulse compressor ( $800 \mathrm{~nm}$, approx. $200 \mathrm{ps}$ ).

After the samples were irradiated, they were characterised in a high-resolution scanning electron microscope (SEM, Zeiss Gemini Supra). For even higher resolution and quantitative analysis of the samples, atomic force microscope (AFM) measurements were conducted on areas of interest (Veeco Multimode with Nanoscope V controller).

Depending on the level of contamination with nanoscopic particles due to redeposition of the ablated species, some samples had to be cleaned before the AFM measurements were carried out. A snow jet cleaning setup was used for the removal of the particles. It utilises the freezing and immediate evaporation of a highly pressurised stream of $\mathrm{CO}_{2}$ onto and from the heated substrate to remove loose particles from the sample surface.

Even though this method has been reported to be very disruptive to surfaces, here we could not find any sign of surface modification (i.e. scratches) that could be linked to the removal process even with large-area SEM/AFM scans.

In order to compare the results with theory, we carried out calculations using finite difference in the time domain (FDTD) simulation software provided by Lumerical Inc. [11]. One unit cell of metallic nanotriangles which form the periodic lattice was calculated by the use of periodic boundary conditions. The rounded edges and slightly curved sides of the triangles were also taken into account. In order to distinguish between the different excitation modes for a given structure, we compared the absorption spectra of gold nanotriangles on a glass substrate between experiment and simulation. In a next step, these nanostructures were transferred onto a silicon substrate to simulate the ablation method. For the elucidation of single-particle effects versus periodic lattice influence, we repeated the same calculations exemplarily for an isolated triangle.

\subsection{Femtosecond ablation}

Femtosecond laser ablation was carried out for two different sizes of gold nanotriangles. When using colloids with a diameter of $320 \mathrm{~nm}$, one obtains triangles with a side length of about $85 \mathrm{~nm}$. Larger triangles with a side length of $540 \mathrm{~nm}$ were produced using colloids with a diameter of $1500 \mathrm{~nm}$ (the colloids were polystyrene particles by Interfacial Dynamics Corp.) [22].

Single shots of femtosecond pulses were applied to homogeneous regions with particle arrays. When looking at the general intensity dependence of the ablation pattern of a single laser pulse (i.e. going from the rim of a single laser spot to higher intensities), both sizes undergo the same stages of interaction between the incident laser light and the nanostructures. The particles will be expelled at rather low fluence and can sometimes be found in places where they have not been deposited. On almost every place where a triangle had been lying, holes appear at certain spots below the triangle, even if a direct neighbour is still at its place. These holes are at first situated close to two of the three tips of a triangle for the polarisation considered in this article. The holes increase in size as the fluence is raised. For the highest fluences, the surface between the triangles is also affected by the laser pulse. Due to the very inhomogeneous intensity profile of our laser system at the time of the experiment, an absolute value for the different fluence regimes could be roughly guessed at best. Therefore, we will limit our discussion to be purely phenomenological. 
Figure 1 exemplifies the evolution of such holes with higher fluences for the larger triangles. Note that for higher fluences an additional hole appears in the previously unaf-

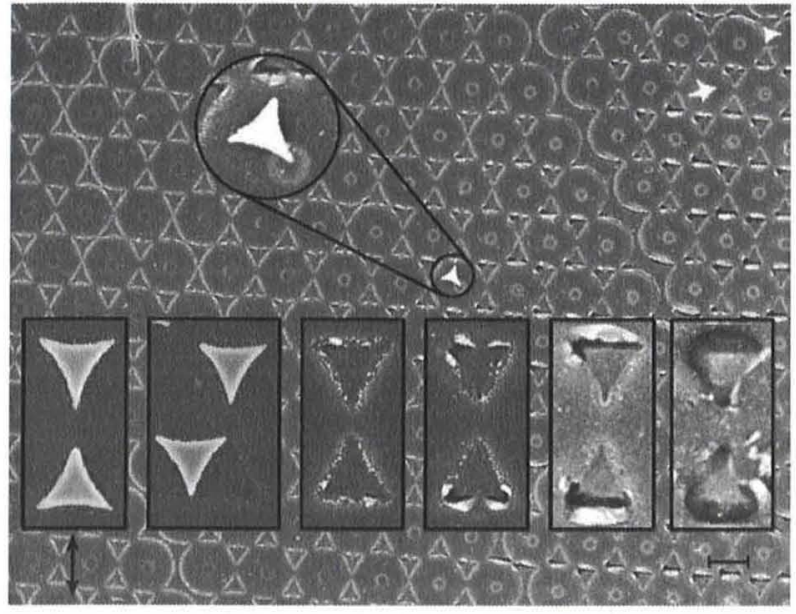

Fig. 1 SEM image showing the evolution of the surface modification by the near field of nanotriangles with a side length of $540 \mathrm{~nm}$ with increasing (from left to right) femtosecond laser fluence. Detail: ablation pattern for pairs of nanotriangles at increasing fluences. Round inset: blow-up of a redeposited single nanotriangle. The double-headed arrow gives the direction of the polarisation of the incident laser light. Scale bar: $1 \mu \mathrm{m}$ fected tip of the triangle. The small droplets that are visible around the former position of the triangle are a by-product of the deposition process and are not connected to the ablation process.

While the general intensity dependence is the same for both sizes of triangles, the distribution of the holes in a medium-fluence regime shows a major difference. Figure 2 presents the ablation pattern at a medium-fluence regime for both sizes.

Looking at the smaller triangles, the holes form on those tips which are aligned parallel to the polarisation of the laser beam. Additionally, they can be found directly below the tips of the structures.

For the larger triangles, the situation is quite different. There, the holes form below those tips that are aligned perpendicularly to the polarisation of the incident laser beam. Furthermore, they can now be found a few nanometres away from the actual tip of the triangle. With increasing fluence, an additional hole appears at the tip of the triangle, which was absent from the ablation pattern with lower fluences (see also Fig. 1).

\subsection{Picosecond ablation}

Picosecond irradiation experiments were carried out on only one size of triangular nanostructures. Colloidal masks con-
Fig. 2 AFM images of the ablation pattern for nanotriangles with a side length of $85 \mathrm{~nm}($ left $)$ and $540 \mathrm{~nm}$ $(r i g h t)$. For the measurement of the smaller triangles, the sample had to be cleaned using the snow jet technique. The position of the height profile is depicted in the AFM image with a bar in the corresponding colour. In the graph, the profiles are shown with an arbitrary offset. The double-headed arrows give the direction of the polarisation of the incident laser light. Scale bars: $100 \mathrm{~nm}$ (left) and $300 \mathrm{~nm}$ (right). False colour rulers: $0 \mathrm{~nm}$ to $10 \mathrm{~nm}$ (left) and $0 \mathrm{~nm}$ to $70 \mathrm{~nm}$ (right)

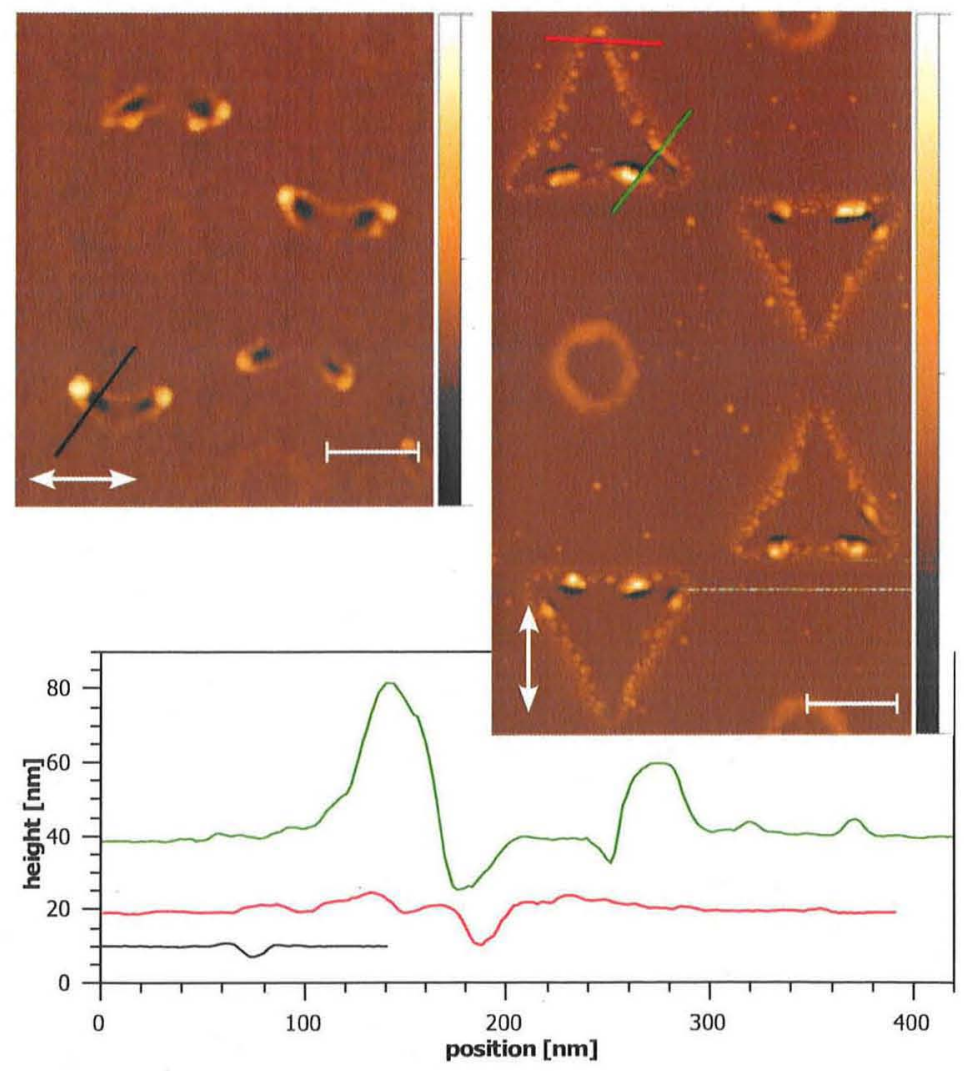


sisting of particles with a diameter of $1740 \mathrm{~nm}$ (polystyrene particles by Microparticles $\mathrm{GmbH}$ ) were used to prepare slightly larger triangles than in the fs experiments. While the direct comparison between the ablation patterns is not possible due to the difference in particle size, a general comparison between the ablation mechanisms should still be feasible.

Looking at the energy dependence for ps ablation, there are more distinguishable regimes than in the case of fs ablation. Furthermore, even though ablation occurs at higher fluences, the patterns obtained are quite different in comparison with fs experiments. Figure 3 shows examples for the various regimes. Coming from low fluences, the triangles deform before they get ablated. In contrast to the melting of nanostructures induced by nanosecond laser irradiation or high repetition rate femtosecond irradiation [23-25], the deformation of the triangle can at first only be seen on two of the three tips, which is strictly reproducible. Figure 4 compares the shape of six different triangles with the two different orientations according to the position in the array. In the background a larger area of modified triangles is shown, demonstrating the uniformity of the process. One can see that even the details of the melting of the tips are reproduced for triangles with the same orientation.

At higher intensities, the whole triangle gets deformed until it is removed without modification of the underlying surface.

With increasing laser fluence, small droplets appear on the surfaces in the regions where the triangles were situated prior to irradiation. Still, no modification of the surface is noticeable.
Upon further increase of the intensity, surface modification can be found. It appears at first at those tips of the triangles that are directed in the same way as those where the first melting could be observed for lower fluences. At the highest fluences, the whole surface below the triangles is modified. For a more quantitative analysis, an AFM image showing the ablation pattern of a single nanotriangle is depicted in the left-hand half of Fig. 3.

\subsection{Simulation/absorption spectra}

Figure 5 shows the calculated field distributions for the triangular structures on silicon used for the fs ablation exper-

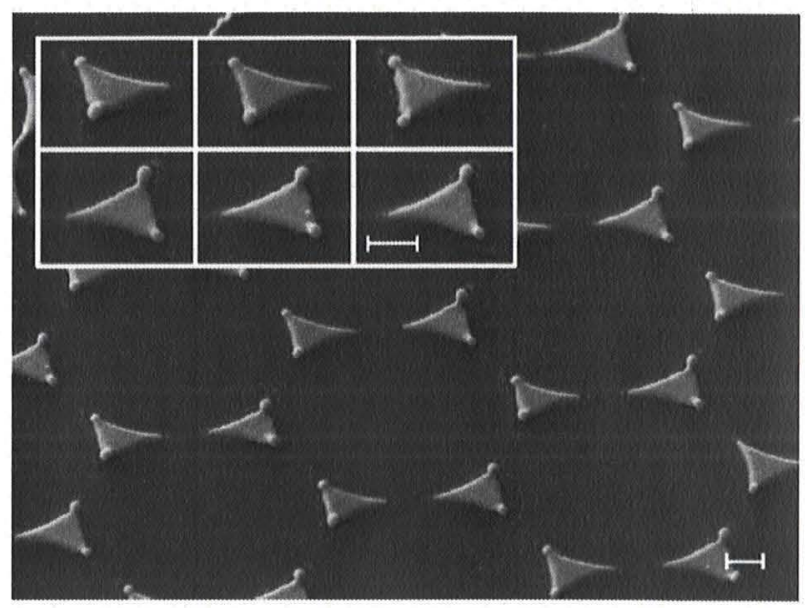

Fig. 4 SEM image of modified nanotriangles (produced with $1740 \mathrm{~nm}$ colloids) after ps laser irradiation. Scale bars: $300 \mathrm{~nm}$
Fig. 3 (Left; a-f) SEM images showing the evolution of the surface modification by the near field of nanotriangles produced by $1740 \mathrm{~nm}$ colloids with increasing picosecond laser fluence. (right) AFM image of the surface modification by picosecond near-field ablation (measurement after snow jet cleaning). The position of the height profile is depicted in the AFM image with a bar in the corresponding colour. In the graph, the profiles are shown with an arbitrary offset. The double-headed arrows give the direction of the polarisation of the incident laser light. Scale bars: $300 \mathrm{~nm}$. False colour ruler: $0 \mathrm{~nm}$ to $13 \mathrm{~nm}$
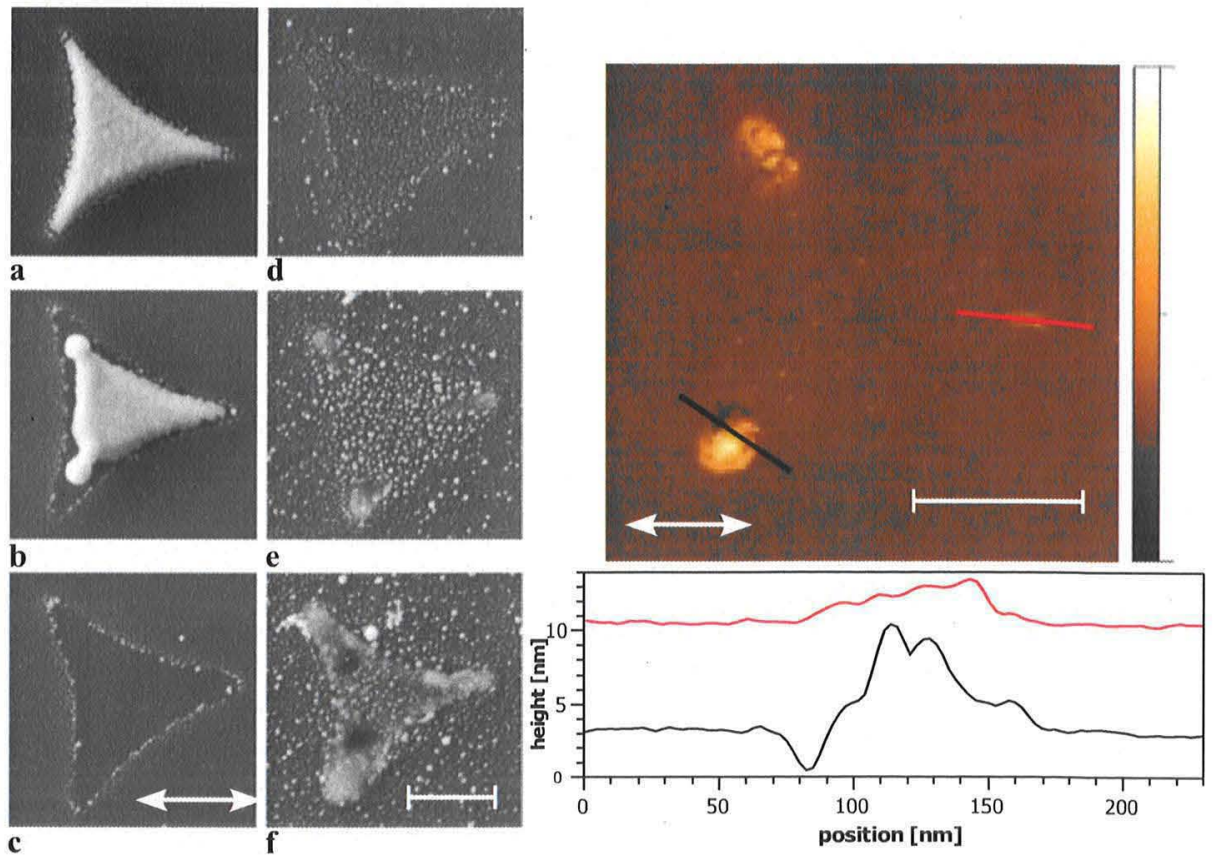
iments. Furthermore, both the calculated and the measured absorption spectra for the same triangles on a glass substrate are shown.

Comparing the calculated field distributions to the ablation patterns in Fig. 2, one can see that the ablation pattern is reproduced in great detail.

The absorption spectra show a good general agreement between experiment and simulation as well. However, in the experimental spectra a much broader distribution and a slight red shift of the main peak are noticeable, especially for the smaller triangles.

For the smaller triangles, distinct peaks at $720 \mathrm{~nm}$ and $760 \mathrm{~nm}$ show up in the simulation and measurement, respectively. For the larger triangles, the main peak lies at $1700 \mathrm{~nm}$ for both the experiment and the calculation. For smaller wavelengths additional peaks are calculated, which mark multipole excitations. The measured spectrum shows only one broad peak in this region, probably due to size and shape variations of the produced structures.

\section{Discussion}

\subsection{Near-field distribution}

Comparing ablation patterns for the smaller and the larger triangles after fs irradiation (Fig. 2), the most striking dif- ference is that the pattern for the larger triangles is tilted by $90^{\circ}$ against the pattern of the smaller triangles with respect to the polarisation of the incident laser light.

While the smaller triangles show a behaviour one would expect from a dipolar antenna, the ablation pattern of the larger triangles contradicts such a simple explanation.

Such tilting of the ablation pattern has been described in earlier publications [13-15] but could not be replicated by discrete dipole approximation (DDA) calculations. To explain the deviation between experiment and calculation, it was assumed that the field of the adjacent triangles influences the local field distribution of each triangle.

With the help of the comparison of the absorption spectra obtained by experiment and simulation, the FDTD calculations (Fig. 5) show almost perfect agreement with the respective ablation pattern. Furthermore, the field distribution does not change if only a single triangular structure is simulated (inset of Fig. 5), indicating that the influence of neighbouring triangles is at best marginal.

To understand the field distribution of the $540 \mathrm{~nm}$ triangles, one has to take the absorption spectra into account. The peak around $800 \mathrm{~nm}$ of the smaller triangles is associated with a regular dipolar resonance of these structures. It can easily be excited by the $800 \mathrm{~nm}$ radiation of the Ti:Sa laser.

For the larger structures, however, the large dipolar peak can be found in the infrared, which makes it impossible to excite with $800 \mathrm{~nm}$ laser light.
Fig. 5 Measured and calculated absorption spectra for $85 \mathrm{~nm}$ (left) and $540 \mathrm{~nm}($ right $)$ nanotriangles on glass with the corresponding calculated scattered field intensities (normalised on the incoming field) for $800 \mathrm{~nm}$ irradiation on silicon. Small inset: calculated field distribution for a single $540 \mathrm{~nm}$ nanotriangle. The double-headed arrows give the direction of the polarisation of the incident laser light. Scale bars: $100 \mathrm{~nm}$ (left) and $300 \mathrm{~nm}$ (right) False colour rulers: 0 to 255 (left) and 0 to 30 (right)
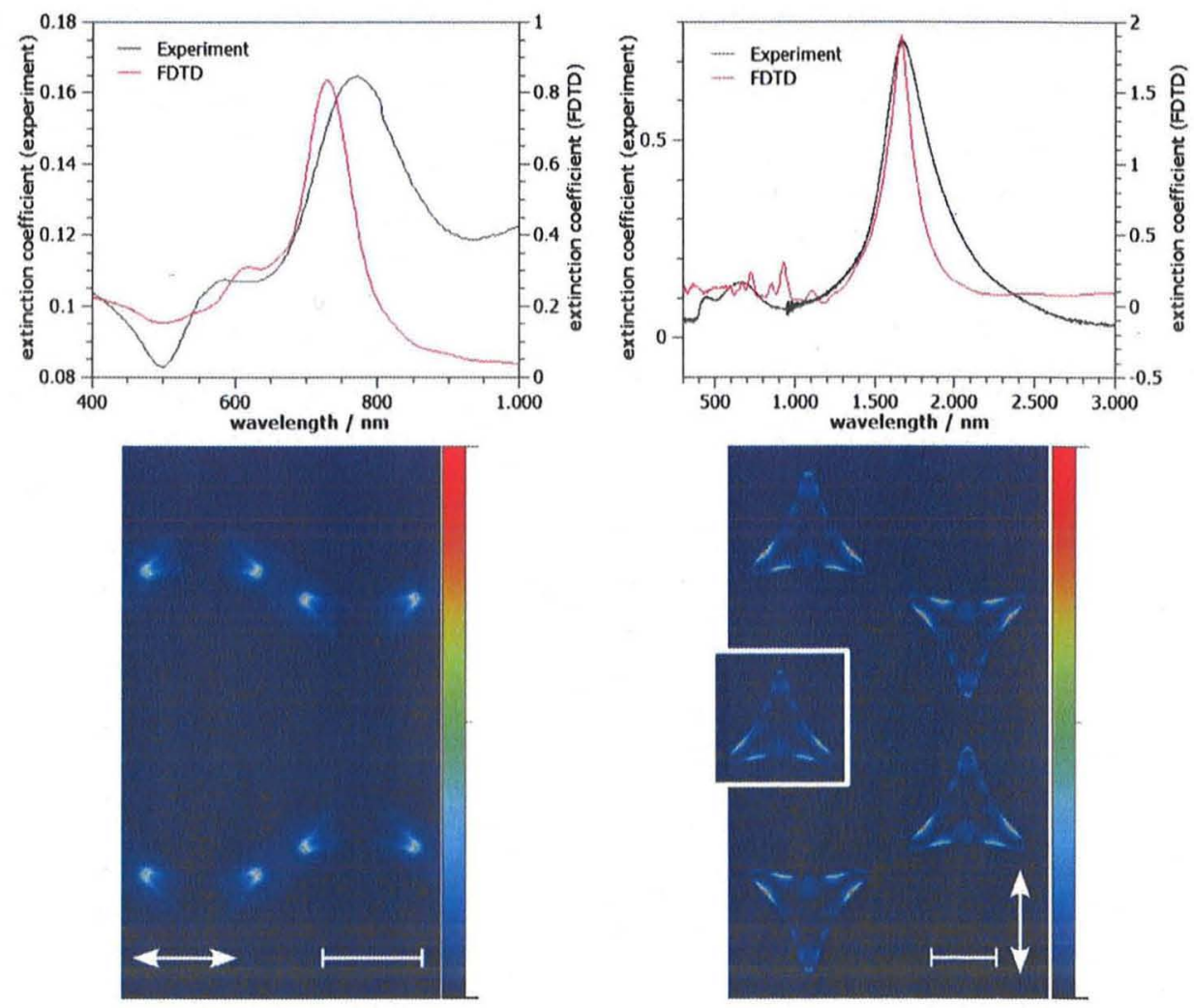
In the vicinity of the laser wavelength, one can see different smaller peaks in the simulation or one broader peak in the measured spectrum. These peaks correspond to quadrupolar excitations of the triangles. Those excitations do have field distributions that are perpendicular to the polarisation of the incident laser light.

\subsection{Comparison between ps and fs ablation}

Ablation is in general a thermal process, where materials get heated to above the spinodal line separating the metastable and unstable parts of the liquid. Femtosecond ablation on semiconductors in particular is discussed in terms of nonthermal processes caused by the strong depletion of carriers from the valence band into the (antibinding) conduction band $[15,26,27]$. This explains the exceedingly low ablation threshold fluence found in semiconductors, such as silicon or germanium.

Here both pictures are complicated moreover by the presence of the gold triangles, which can undergo a phase transition as well. With femtosecond irradiation the triangles are completely expelled from the surface, leaving behind an ablation crater in the substrate. For the picosecond pulses the ablation on the substrate is much less pronounced due to the smaller absolute amplitude of the field. This implies at the same time that the ablation process on the substrate is indeed of non-thermal origin.

With ps irradiation, a clear local deformation of the triangles can be observed (note that the orientation, given by the deformed tips of the triangles, is perpendicular to the polarisation of the laser). Taking into account the rounded shape of the deformed triangles and comparing it to ns melting experiments [23], this deformation can be associated with nanoscale confined melting. In its liquid state, the surface tension of the gold leads to the local formation of a droplet still connected to the triangle.

The main difference to earlier ns and high repetition rate fs melting experiments conducted on nanotriangles [23-25] is the local confinement and selectivity of the ps melting. While the melting of only the tips of the nanoscopic triangular structures has been reported in previous publications [23-25], the reproducible, selective melting of only two of the three tips of the triangles has not been observed. Furthermore, as in the fs ablation experiments, the direction given by the molten tips of the triangles is perpendicular to the laser polarisation. All of this is strong evidence suggesting that the triangle is heated locally due to the strong near-field enhancement. However, in the ps time scale, the thermal diffusion length

$L_{\mathrm{D}}=\sqrt{D \cdot \tau}$

(with $\tau=$ pulse length and $D=$ diffusion coefficient) is still too small to affect the whole structure $\left(L_{\mathrm{D}}(200 \mathrm{ps} ; \mathrm{Au})\right.$ $\sim 160 \mathrm{~nm} ; L_{\text {Triangle }}>500 \mathrm{~nm}$ ). Thus, nanometre confined heating is achieved.

However, such partially melted structures have never been observed in single-pulse fs ablation experiments. What can often be found instead are still intact gold triangles that are redeposited on the surface after ablation (see blow-up in Fig. 1) [24, 25].

Comparing the regions with strong surface modification for fs and ps ablation for larger triangles (right-hand halves of Figs. 2 and 3) also shows large differences in the two time domains. While the fs ablation patterns reproduce the calculations in a very detailed fashion, the ps patterns show only blurred features. Additionally, small hills can be found on the silicon surfaces below the triangle. Comparing the height profiles for both time regimes, one can see that while there are relatively deep holes with quite high residues at the rim for the fs ablation, the surface modification is much more delicate in the case of ps irradiation. Here, a relatively shallow hole with a relatively flat hill at the rim is found below the triangle's tip. In contrast to the fs experiments, however, the whole area below the tip seems to be affected.

Taking all these observations into account, the most likely process for ps surface modification seems to be melting of the silicon in the region of the strongest near fields. During the following fast-freezing process, the silicon will be stuck in a polycrystalline or amorphous state, explaining the formation of the aforementioned hills. The more blurry features in the case of the ps imaging supports this explanation, as this can be attributed to the smoothing effect of a melting phase.

\section{Conclusions}

This study has discussed the phenomenology of structural evolution with femtosecond and picosecond ablation at nanotriangle-decorated silicon surfaces. The near-field enhancement of the plasmonic gold nanostructures creates very localised ablation structures that may be very useful for non-contact patterning of surfaces. For a better understanding of the ablation process, a comparison between the ablated structures and near-field distributions from FDTD calculations has been made. Not only a qualitative, but also a quantitative agreement has been found for the a priori astonishing finding that the enhancement and thus ablation efficiency is not simply located at the triangle tips. Depending on the triangle sizes, ablation maxima along the triangle edges are observed. The FDTD calculation correctly assigns these patterns as multipole excitation of the triangles. This explains the strong difference between the smaller particles, where the laser wavelength is close to the primary (dipolar) resonance, while this resonance shows a red shift with size, letting the laser wavelength coincide with higher multipole excitation. 
Picosecond pulses can also cause ablation on the substrate, but only at higher fluence, coincident with the nonthermal nature of the ablation in silicon. At the same time, a rich morphology change in the nanotriangles themselves becomes apparent. As energy deposition is still confined, the melting of the triangles can be initiated selectively at those tips which show the largest field enhancement.

For a deeper understanding of the underlying processes, quantitative measurements of the different regime thresholds are necessary and are presently being carried out. They will provide a quantitative determination of the near-field enhancement in the maxima of the field distribution. Even though near-field effects are being used for a large number of different applications, direct measurements of the absolute field enhancement are rather sparse until now.

Acknowledgements We thank the Schwerpunktprogramm (SPP) 1327 of the DFG and the Strategic Japanese-German Cooperative Program of the JST and DFG for their financial support.

\section{References}

1. H.-J. Muenzer, M. Mosbacher, M. Bertsch, J. Zimmermann, P. Leiderer, J. Boneberg, J. Microsc. 202, 129 (2001)

2. J.N. Farahani, D.W. Pohl, H.-J. Eisler, B. Hecht, Phys. Rev. Lett. 95, 017402 (2005)

3. S. Nie, S.R. Emory, Science 275, 1102 (1997)

4. A.D. McFarland, M.A. Young, J.A. Dieringer, R.P. Van Duyne, J. Phys. Chem. B 109, 11279 (2005)

5. T. Hanke, G. Krauss, D. Traeutlein, B. Wild, R. Bratschitsch, A. Leitenstorfer, Phys. Rev. Lett. 103, 257404 (2009)

6. J.A. Schuller, E.S. Barnard, W. Cai, Y.C. Jun, J.S. White, M.L. Brongersma, Nat. Mater. 9, 193 (2010)

7. R. Hillenbrand, F. Keilmann, P. Hanarp, D.S. Sutherland, J. Aizpurua, Appl. Phys. Lett. 83, 368 (2003)

8. P. Kuehler, F.J. Garcia de Abajo, J. Solis, M. Mosbacher, P. Leiderer, C. Afonso, J. Siegel, Small 5, 1825 (2009)
9. J. Siegel, D. Puerto, J. Solis, F.J. Garcia de Abajo, C.N. Afonso, M. Longo, C. Wiemer, M. Fanciulli, P. Kuehler, M. Mosbacher, P. Leiderer, Appl. Phys. Lett. 96, 193108 (2010)

10. C. Hubert, A. Rumyantseva, G. Lerondel, J. Grand, S. Kostcheev, L. Billot, A. Vial, R. Bachelot, P. Royer, S. Chang, S.K. Gray, G.P. Wiederrecht, G.C. Schatz, Nano Lett. 5, 615 (2005)

11. K. Ueno, S. Juodkazis, T. Shibuya, Y. Yokota, V. Mizeikis, K. Sasaki, H. Misawa, J. Am. Chem. Soc. 130, 6928 (2008)

12. N. Murazawa, K. Ueno, V. Mizeikis, S. Juodkazis, H. Misawa, J. Phys. Chem. C 113, 1147 (2009)

13. P. Leiderer, C. Bartels, J. Konig-Birk, M. Mosbacher, J. Boneberg, Appl. Phys. Lett. 85, 5370 (2004)

14. J. Boneberg, J. Koenig-Birk, H.-J. Muenzer, P. Leiderer, K.L. Shuford, G.C. Schatz, Appl. Phys. A, Mater. Sci. Process. 89, 299 (2007)

15. A. Plech, P. Leiderer, J. Boneberg, Laser Photonics Rev. 3, 435 (2009)

16. F. Burmeister, C. Schaefle, T. Matthes, M. Boemisch, J. Boneberg, P. Leiderer, Langmuir 13, 2983 (1997)

17. H.W. Deckman, J.H. Dunsmuir, Appl. Phys. Lett. 41, 377 (1982)

18. B. Keilhofer, C. Bechinger, J. Boneberg, P. Leiderer, F. Burmeister, C. Schaefle, Adv. Mater. 10, 495 (1998)

19. A. Kosiorek, W. Kandulski, P. Chudzinski, K. Kempa, M. Giersig, Nano Lett. 4, 1359 (2004)

20. A. Kosiorek, W. Kandulski, H. Glaczynska, M. Giersig, Small 1, 439 (2005)

21. M.C. Gwinner, E. Koroknay, L. Fu, P. Patoka, W. Kandulski, M. Giersig, H. Giessen, Small 5, 400 (2009)

22. T. Geldhauser, S. Ikegaya, A. Kolloch, N. Murazawa, K. Ueno, J. Boneberg, P. Leiderer, E. Scheer, H. Misawa, Plasmonics 6, 207 (2011). doi:10.1007/s11468-010-9189-9

23. A. Habenicht, M. Olapinski, F. Burmeister, P. Leiderer, J. Boneberg, Science 309, 2043 (2005)

24. W. Huang, W. Qian, M.A. El-Sayed, J. Am. Chem. Soc. 128, 13330 (2006)

25. W. Huang, M.A. El-Sayed, Eur. Phys. J. - Spec. Top 153, 223 (2008)

26. C.V. Shank, R. Yen, C. Hirlimann, Phys. Rev. Lett. 50, 454 (1983)

27. K. Sokolowski-Tinten, J. Bialkowski, D. von der Linde, Phys. Rev. B, Condens. Matter 51, 14186 (1995) 